\title{
Keyword index to volume 36
}

$\alpha$-1-microglobulin 33

4-Aminopyridine 6, 147

Abdominal pain 63

Abnormalities 647

Absorptiometry 647

Action potentials 548

Activities of daily living

(ADL) 485, 491

Acute myelopathy 71

Acute spinal cord injury 683

ADL-index 691

Adverse effects 633

Aerobic training 240

Age 299

Aging 320

Albendazole 67

Alkaptonuria 523

American Spinal Injury Association (ASIA) motor score 491

Ankylosing spondylitis 349

Ankylotic spondylitis 377

Aortic aneurysm complications 110

Aortic laceration 110

Aortic surgery complications 110

Apoptosis 683

Arachnoid cyst 864

Arm cranking 750

Arm-cranking ergometry 205

Arm cranking exercise 579

Arm exercise 691

ASIA motor score 621

ASIA score 380

ASIA/IMSOP standards 554

Assessment 427

Astrocyte 811

Atlanto-axial 661

Atlantoaxial subluxation 520

Autonomic dysreflexia 756, 761

Avulsion 391

Axonal regrowth 391

Axon regeneration 811

Baclofen 699, 854

Balanced bladder 476

Behavior 724

Biochemical markers 822

Biphasic positive airway pressure system (BiPAP) 481

Bisacodyl 777

Bladder neck 345

Blood pressure 691

Body composition 463, 637
Bone 637, 647

Bone density 641

Bone methabolism 641

Bone mineral density (BMD) 822

Bone wire 797

Botulinum toxin 91

Botulinum toxin type A 854

Bowel dysfunction 485

Brachial plexus 391

Brachial plexus lesions 588

Brain death 741

Breast screening 340

Brown-Sequard Syndrome 797

Camel collision injuries 415

Capsaïcin 95

Cardiac output 691

Care study 443

Catheter 177

Catheterization 171, 299

Central cord syndrome 156

Central neuropathic pain 601

Cerebral complications 732

Cerebral palsy 288

Cervical disc herniation 288

Cervical myeloradiculopathy 399

Cervical screening 340

Cervical spinal cord injury 491, 567

Cervical spine 21, 859

Cervical spondylitic myelopathy 629

Cervical spondylosis 156

Cervico-thoracic spine 561

CFES LE 782

CHD risk 750

Child 864

Childhood onset 181

Cholesterol 228

Choriocarcinoma 366

Chronic pain 671

Chronic spinal cord injury 6

Chronic ventilatory failure 310

Classification 205, 554

Clavicle fracture 797

Cleavage 177

Clinical outcome 171

Clonidine 699

Coagulopathy 658

Cognitive impairment 231

Colon 777

Colon transit 847

Communication system 104

Computed tomography 661

Computer modelling 531
Conservative and surgical treatment 567

Constipation 63, 777, 847

Continent ileocecal augmentation 246

Contractures 405

Coronary heart disease 13

CT scan 137

Cyproheptadine 699

Cysticercosis 285

Cystoplasty 246

Data base 57

Degenerative 186

Dejerine 78

Dejerine-Klumpke 78

Dens 661

Densitometry 647

Detraining 782

Detrusor hyperactivity 95

Detrusor hyperreflexia 100

Detrusor pressure 171

Detrusor-sphincter dyssynergia 2, 39, 91

Developing country 353

DEXA 637

Diaphragmatic pacemaker 310

Disc prolapse 523

Discharge planning 262

Dislocation 21

Divorce 252

Dog reimplatation 391

Dorsal column stimulation 531

Dorsal root stimulation 531

Double-crush syndrome 399

Down syndrome 520

Dual energy X-ray absorptiometry (DEXA) 822

Dyslipidaemias 228

Dysreflexia 756

Echinococcus 166

Ejaculation 132

Elbow 418

Elbow extension, opponens transfer 315

Electric stimulation 303

Electrical stimulation 100, 750, 847

Electro-acupuncture 476

Electroejaculation 132

Electrophrenic ventilation 310

Electrorectogram 548

Energy consumption 223

Energy cost 223

Entrapment neuropathy 399 
Epidemiology 117, 275

Epidural cooling 654

Epidural electrode 531

Epidural hematoma 71

Epidural stimulator 190

Etiopathological 186

Evaluation 832

Evoked potentials 497

Exercise 18

Extracorporeal shockwave lithotripsy (ESWL) 454

Extradural 285

Faecal incontinence 63, 774

Fat 637

FES 443

Finger flexion transfer 315

Follow-up studies 45

Fracture 593, 790

Fracture-dislocation 377

Fracture of cervical spine 377

Frankel grading scale 491

Functional assessment 832

Functional electrical stimulation 463

Functional programmes 514

Functional status 507

Functioning 252

Gait 699

Gastrointestinal problems 485

Gaze 104

Genital flora 617

Geriatric 629

Glucose intolerance 228

Greater sciatic notch 584

Haemophilia 658

Halo device 732

Halo immobilization 520

Hand positioning 818

HDL cholesterol 228

Health behavior 724

Health promotion 724

Health-related quality of life 193

Health services 280

Hip 647

Historical review 78

Hydatid cyst 166

Hypoxia 231

Iatrogenic hypospadias 177

Incidence 574,790

Incontinence 777

Independence 427

Infusion pumps 854

Intermittent catheterization 29 , 409

Intermittent catheterization program 476
Intradural extra-arachnoid tumour 859

Intramedullary cysticercosis 67

Intrathecal injection 497

Invasive aspergillosis 800

Immunocompetence 800

Iodine overload 593

Ischaemic myelopathy 110

Isokinetic dynamometry 716

Isometric contraction 463

Kidney stones 454

Laminectomy 200

Laufband 744

Lean tissue mass 637

Level of lesion 818

Life adjustment 51

Life conditions 117

Life expectancy 329

Life situation 213

Lipids 13

'Locked-in' syndrome 741

Locomotion 744

Locomotor training 380

Long-term outcome 117

Long term quality of life 87

Lower limb impairments 205

Lumbar spinal stenosis 200

Magnetic resonance imaging 137 , $363,658,661$

Magnetic resonance imaging (MRI) 71

Management of complication 377

Manipulation under general anaesthesia 21

Manual muscle test 716

Marital adjustment 826

Marital satisfaction 826

Marital stability 826

Mechanical stress 186

Medical follow-up 117

Medical services 514

Medications 161

Melphalan 470

Memokath stent 39

Men 337

Metastases 366

Methylprednisolone 6, 633

Mobility 427

Monoamines 18

Morbidity 213

Mortality 213, 266, 275, 329, 574

Motion 418

Motor evoked potential 147, 380

Multiple myeloma 470

Multiple sclerosis 95

Muscle atrophy 463
Muscle strength 691

Musculocutaneous nerve 588

Musculocutaneous nerve palsy 591

Mycobacterium tuberculosis 584

Myeloma 661

Myelopathy 67

Myometry 716

National survey 280

Nerve entrapments 588

Nerve growth factor receptor 541

Neuroanatomy 78

Neurocysticercosis 67

Neurogenic bladder 476

Neurological complications 470

Neurological outcome 353

Neurologic situation 818

Neuronal death 683

Neuropathic bladder 2, 33, 39, 95, 246, 345, 409, 541

Neuropathic bowel 485

Neuroprosthetics 847

Neurostimulation 671

Nimodipine 497

NMES 443

Non-compliance 262

Nurick's criteria 629

Ochranosis 523

Oligodendrocyte 811

Orthosis 223

Orthostatic hypotension 87

Ossification 647

Ossification of the posterior longitudinal ligament 561

Ossification of the yellow ligament 561

Osteonecrosis 633, 641

Osteoporosis 641

Osteoporosis-spinal cord injury 822

Outcomes 45, 320

Oxybutynin 409

Oxygen desaturation 621

$\mathrm{P}^{75}$ receptor 541

Pacing 310

Pacesetter potentials 548

Paediatric spine 732

Pain 25

Paraplegia 13, 18, 95, 125, 228, 240, 303, 329, 337, 366, 405, $427,613,637,790,800$

Paraplegic 223, 579, 744, 750

Partner relationship 252

Pathophysiology 601

Peak oxygen uptake 579

Pediatric spinal cord injury 193, 641

Pelvic ganglia 548 
Penile erection 125

Persistent vegetative state 741

Pharmacokinetics 147

Physical activity 205

Physical fitness 205

Physical therapy 427

Physiotherapeutical measures 818

Physostigmine 132

Plasmacytoma 661

Poliomyelitis 280

Polytrauma 800

Post-traumatic canal stenosis 137

Post traumatic paraplegia 432

Post-traumatic syringomyelia 137

Pressure sores 432, 593

Pressure ulcer 443

Prevention 415

Primary spinal cord melanoma 363

Prognosis 567

Prostate cancer 299

Prostate specific antigen 33, 771

Psychology 181

Psycho-social aspects 262

Pudendal nerve 100

Pulmonary function testing 240

Pyelonephritis, paraplegia 33

Pyogenic spinal infection 507

Quadriplegia 832

Quadriplegic 750

Quality of life 193, 320

Quality of marital life 826

Radiological 186

Range of motion 418

Rats 6

Readmission 838

Reconstruction 177

Rectum 548

Recurrent infections 613

Reduction 21

Registry 57

Rehabilitation 156, 240, 280, 432, 514, 574, 724

Rehabilitation outcome 507

Remodelling of spinal cord circuits 761

Reproduction 132

Resection 345

Respiratory support 481

Retrograde continence enema 774

Risk factor 790

Satisfaction 171

SCI 63

Schwann cells 811

Schwannoma 859

Sciatic nerve palsy 584
Scoliosis 732

Secondary conditions 45, 337

Secondary damage 654

Secondary injury 683

Self-care 432

Self-perception 181

Semen 771

Seminal plasma 771

Semiology 78

Serotonin 18

Sexuality 252

Shoulder 418

Skin sores 51

Sleep 621

Sleep apnea syndrome 621

Sleep apnoea 231

Slow waves 548

Solitary plasmacytoma 470

Spasticity 147, 156, 190, 854

Spina bifida 177, 613, 774

Spinal arachnoid cyst 864

Spinal compression 285

Spinal cord 67, 71, 78, 299, 363, 744, 782, 811

Spinal cord blood flow 497

Spinal cord compression 166, 366

Spinal cord disease 91

Spinal cord dysfunction 57

Spinal cord injured couples 826

Spinal cord injuries 25, 137, 193, 252, 320, 613, 691, 699, 771

Spinal cord injuries hospital 514

Spinal cord injury 18, 29, 33, 39, 45, 51, 57, 117, 125, 147, 161,

$177,181,190,205,213,240$,

262, 266, 275, 288, 315, 337,

345, 353, 405, 409, 415, 432,

454, 463, 476, 481, 485, 497,

554, 574, 579, 591, 601, 617,

633, 641, 654, 716, 756, 761,

777, 790, 797, 832, 838, 847, 854

Spinal cord injury

complications 45

Spinal cord injury patients 100

Spinal cord injury (SCI) 186, 340

Spinal cord stimulation 531, 671

Spinal cysticercosis 285

Spinal epidural hematoma 349

Spinal injury 132

Spinal locomotor pattern 380

Spinal nerve roots 303

Spinal trauma 864

Spinal tuberculosis 507

Spinal tumors 363

Spine 470, 523

Spine fracture 349

Spondylodiscitis 800

Spondylolisthesis 200
Spontaneous spinal epidural haematoma 658

Staff 514

Standardised mortality ratio 329

Stents 39

Suicide 262

Suppository 777

Suprapubic catheters 171

Surgery 520, 561, 629

Survival analysis 266, 275

Survival rate 329

Sympathetic skin responses (SSR) 125

Tendon transfers 315

Terazosin 761

Tetraplegia 13, 71, 87, 104, 117, 186, 228, 231, 310, 329, 377, 405, 443, 454, 613, 621, 741, 761,838

Tetraplegic 744

Tetraplegic hand function 818

Therapy 601

Thoracic spine 561

Tibial nerve somatosensory evoked potential 380

Transurethral sphincterotomy 2

Traumatic brain injuries 252

Traumatic spinal cord injury 349

Treadmill 744

Trophic factors 811

Tuberculous abscess 584

Upper urinary tract dysfunction 29

Urinary infection 454

Urinary tract infection $33,39,432$, 617

Urinary tract infections precipitating antibodies 613

Urodynamics 29, 345, 756

Ventral root 391

Vertebral hydatid cyst 166

Vibratory stimulation 132

Walking 223

Wheelchair 418

Wheelchair propulsion 418

Wheelchair sport 579

Women 337

Wrist extension 315

Young children 520

Zancolli's classification 491

Zimbabwe 213 